Research Article

\title{
An Improved Channel Access Model for IEEE 802.11 WLANs
}

\author{
M. Ekpenyong ${ }^{*}, 1$ and E. Inyang ${ }^{2}$. \\ ${ }^{1}$ School of Informatics, University of Edinburgh, Edinburgh EH8 9AB, UK, \\ ${ }^{2}$ Dept of Computer Science, University of Uyo, PMB. 1017, Uyo 520003, Nigeria
}

Received 30 September 2012; Accepted 7 November 2013

\begin{abstract}
In this paper, a robust channel access strategy is proposed. The research is motivated by the inefficiency of current IEEE 802.11 WLAN systems. We adopt a dynamic allocation strategy that improves throughput efficiency in high-data-rate WLANs, by splitting the packet transfer of the system to speedup communication. The proposed model grants each node autonomous access to the pool of available resources using orthogonal frequency division multiplexing (OFDM) - a fourth generation wireless technology. The models for the system are derived and simulated with field data for both inbound and outbound traffics. Results obtained from the simulation represents best practices and yields better system performance compared to the existing IEEE 802.11 WLAN systems.
\end{abstract}

Keywords: Network efficiency, data throughput, capacity and performance, fine-grained model.

\section{Introduction}

In a wireless local area network (WLAN) deployment, performance and capacity are mostly influenced by several interacting factors necessary to fulfill the requirements of high availability, high performance, efficiency and missioncritical environment. Most of the IEEE 802.11 technological innovations so far aim at increasing the range and coverage of WLANs; and for effective QoS, WLANs should be designed to enable wireless access equipment offer the expected coverage with sufficient capacity to bear the expected load. Research studies on operational WLANs have shown that the traffic load is often distributed unevenly among access points (APs). Thus, the AP location and frequency allocation are two important aspects in an optimized WLAN design. AP position should first be based on the actual needs of the existing system and then adjusted through field measurements. Given the dynamic nature of users demand, the load capacity of an AP may either increase or decrease. These changes can be observed using a WLAN monitor. Also, interference from collocated wireless devices operating within the same unlicensed frequency band may lead to instability of WLANs, resulting in frequent failures of the access points. Therefore, noise and interference, signal quality, transmission rate and device configuration greatly affect the performance of WLANs. In a dynamic operational WLAN environment, interference may hamper the signal quality, hence impacting negatively on the network management decision making. The ability of the system to deal with such dynamic changes and move from a previous stable state to a new optional stable state in quick

\footnotetext{
*E-mail address: ekpenyong_moses@yahoo.com

ISSN: 1791-2377 @ 2011 Kavala Institute of Technology. All rights reserved.
}

succession becomes an issue.

The wireless medium is a dynamically shared device affected by several interacting factors, some of which can be controlled, while others are fundamentally limited by the wireless medium. These limitations must be recognized and taken into account when planning a WLAN. Modern communication technologies are constantly advancing the physical (PHY) data rates in WLANs. This growth in capacity is achieved mostly through wider channel bandwidths and advanced PHY techniques like multipleinput multiple-output (MIMO). Other standards like IEEE $802.11 \mathrm{ac}$ and 802.11 ad are determined to provide even faster PHY rates (>1Gbps). However, the data throughput efficiency - the ratio between the network throughput and the PHY data rate, degrades rapidly as the PHY data rate increases due to the design of current 802.11 medium access control (MAC) protocol [1]. The fundamental reason for this inefficiency is as a result of full allocation of the entire channel to one station as a single resource, by current MAC devices.

This paper therefore studies and evaluates the performance of WLANs. We concentrate on network efficiency and verify the system performance of an existing WLAN that provides Internet services to customers. A finegrained solution which improves on the performance of the existing system is proposed and the performance of the proposed model is validated against the existing system's performance.

\section{Related Works}

Recently, the IEEE 802.11 WLAN has been widely deployed with detailed specification of both MAC and PHY 
and the most researched area being the MAC layer [2]. One way to improve the MAC efficiency is to extend the channel time for data transmissions by sending larger frames. The IEEE $802.11 \mathrm{n}$ permits frame aggregation, i.e., transmits multiple frames simultaneously in one contention window. Larger aggregated frames however results in longer delays as the sender must wait to collect substantial frames before transmission, thus adversely affecting the TCP and other real-time applications such as voice over internet protocol (VoIP), video conferencing, online chatting, etc. The best way to improve WLAN efficiency is therefore to effectively reduce the channel width and create more channels with widths that meet the PHY data rate and frame size requirements. Multiple stations can then compete and use these channels simultaneously given the traffic demands, thereby reducing the MAC coordination and increasing overall efficiency.

Network efficiency techniques have been extensively covered in literature. In this section, we discuss these techniques under three broad categories. These techniques ensure the improvement of network efficiency in wireless networks without adverse effects on the QoS. The techniques slightly degrade the QoS for individual flows, leading to a significant improvement on the overall system performance:

The wireless stealth multicast technique: In this technique, a minor amount of packet delay is used to detect and eliminate redundancy in the network. This technique represents an extension of the work done in [3], and adopts stealth multicast to the wireless domain. Redundancy in the wireless network is detected at the base station and condensed into a broadcast for transmission across the wireless domain. The redundancy is however eliminated by building virtual multicast groups out of the wireless clients receiving the redundant data. This happens dynamically without changes to the server or client applications. While multicast may not occur all the time, the study done in [4], shows that multicast amenable traffic is most likely to occur during peak periods of usage and the maximum throughput of 802.11 protocols is theoretical, as the achievable maximum throughput falls significantly short in practice. The reason for this is due to link quality, retransmissions and connection on the wireless medium. Wireless stealth multicast improves the quality of service for a congested wireless network in several ways. Firstly, the bandwidth consumed by streaming applications when multiple clients are accessing the same data is reduced, thus enabling more bandwidth to be available for other transmissions. Secondly, since broadcasts do not have a medium access control (MAC) layer acknowledgement, there are less queuing delay between successive transmissions. The works of [5], concentrates on extending IP multicast from the wired domain unto the wireless network which are only related to wireless stealth multicast. Their research aims at increasing the efficiency of multicast transport over the wireless network.

The jumbo gen technique: This technique uses delay to build jumbo grain sized packets which reduces the amount of overhead consumed in the network and allow routers with the heaviest loads to route significantly less number of packets. This allows for greater network scalability and improves the overall performance. In [6], a similar principle is used, but focus is mainly on aggregating TCP acknowledgement with the same destination (into the same packet). Other research works which also focus on router performance include [7-8]. These works introduce new routing techniques in an effort to ensure high-speed performance. Although they fail to address the core problem of increasing line speeds when the average packet size is unscaled, jumbo gen addresses this problem by allowing packet sizes to scale closely with increased line speed. Hence, the number of packets needed to be processed by the core routers remains consistent as the line speed increases. It is important to be aware of the implications high-speed networks and packet aggregation has on TCP performance, as most traffic transferred over the Internet is TCP based. TCP performance in high speed networks has been studied in great depth in [9].

The energy and bandwidth efficient wireless ( $m, k$ ) scheduling (MKR) technique: The MKR technique focuses on trading QoS in terms of packet loss, for an overall improvement of network performance. MKR represents a novel way of conserving energy and bandwidth in the wireless medium. It transmits only the minimum number of packets that ensure an acceptable QoS to end users. Typically, the amount of acceptable loss for end users can be specified using an $(\mathrm{m}, \mathrm{k})$ scheduler. The $(\mathrm{m}, \mathrm{k})$ scheduler is a scheduling technique that has been well studied in real-time systems, in terms of CPU scheduling [10-12], and packet scheduling for the network [13-15]. This is achieved by using the information contained within the $(\mathrm{m}, \mathrm{k})$ parameters with the inclusion of a third parameter, $r$, to proactively drop packets from the stream in order to conserve energy and efficiency in the wireless network, while still providing a reasonable guarantee (provided by $r$ ) that the $(\mathrm{m}, \mathrm{k}$ ) parameters will be met. The overall goal of the MKR packet scheduling is to provide a reasonable level of guarantee of successfully, timely transmission and the minimum number of packets specified by the (m, k, r) parameters.

Gutwin, Fedak, Watson, Dyck and Bell [16], have proposed an alternative approach to improving efficiency, called general message compressor (GMC). According to them, sending messages across network is the means by which the groupware communicates. The messages has different formats (e.g., XML, Plain text, or serialized objects) with different advantages and are extremely inefficient, since they occupy large amount of space to represent small amount of information (i.e., they are verbose) and which overloads the system network resources. Efficiency could be improved by using a more compact format, but this will come at the cost of increased complexity, reduced convenience and reduced readability. The GMC is an automatic compression system that transparently minimizes verbose formats. It does this by automatically finding and removing redundancy in message streams without any knowledge of the content or structure of the message and without any need for the programmer to change the way they work. This is achieved by building a dictionary of sequences that are repeated across a set of messages and replacing sequences with short look-up codes. The GMC compress message data uses a Ziv-Lempel algorithm and Huffman coding [17, 18], and contributes immensely by allowing groupware programmers to use representations that are convenient without sacrificing transport efficiency. The network performance of groupware has many performance defects due to network issues such as latency (the time required for information to travel between locations), jitter (the variance in latency), loss (which results from network packets not arriving at their destination) and insufficient bandwidth. The effect of network delay on users cause collaboration break-down. However, bandwidth 
restriction is one of the most critical causes of latency in distributed systems and the size and efficiency of groupware messages plays a major role in an attempt to improve groupware performance.

Nagurney [19] demonstrates how a network performance efficiency measure called the Nagurney-Qiang (N-Q) measure that captures demands, flows, as well as the behavior of users of the network and incurred costs, can be applied to assess the efficiency of critical infrastructure networks such as transportation networks, the Internet and their network components; and the nodes and links ranking. The result regarding the importance of network components as determine by the N-Q measure are compared to an existing measure proposed in [20], also referred to as the L$\mathrm{M}$ measure. The N-Q measure is a unified measure that can be applied to any network component and is independent of whether the removal of links or nodes would cause a disconnection of the network or not. The L-M measure was proposed to measure the efficiency of networks in which the links may have associated weights or costs.

In this paper, we propose a fine-grained channel access model that improves throughput efficiency in high-data-rate WLANs. We divide the channel width into appropriately sized sub-channels, commensurate with the PHY data rate and typical frame size, and use the orthogonal frequency division multiplexing (OFDM) on the whole channel to avoid bandwidth wastage on guard bands. The fundamental challenge with this approach is coordinating random access among multiple distributed and asynchronous nodes in a WLAN (potentially with multiple APs) without resorting to cellular-style tight timing synchronization. Since coordination in a WLAN is de-centralized in nature, it is impracticable to have OFDMA-style global time synchronization. Not only will this introduce great system complexity, but will also likely require new hardware functionalities beyond the current or emerging 802.11 standards. Furthermore, OFDM does not support random access and hence cannot be directly implemented on a WLAN. Instead, we shall use existing 802.11 coordination mechanisms, such as carrier-sensing and broadcast, to establish rough symbol alignment among contending senders. We shall implement an event-driven simulator to study the performance of a large-scale wireless network and compare its performance side-by-side with the existing system. The simulator will also possess the capability of monitoring both CSMA MAC and OFDM PHY that supports multiple sub-channels. We shall first study and analyze the performance under a coordinating AP network with varying number of stations monitored by a centralized system called the Packeteer and assume that only collisions will cause frame reception failures. Then we concentrate on the uplink transmissions with the application of various traffic patterns with high data rates.

\section{Analysis of IEEE 802.11 WLANs - A case study}

In this research, we obtained field measurements from an existing WLAN operator. The required data were captured using a device called Packet-shaper (Packeteer). This device is operated centrally in the server room of the company where other network and transmission devices are connected. The Packeteer has a life Internet protocol (IP) address which connects and monitors clients. It also has a secured system that requires administrative rights and privileges before login. The system is capable of displaying reports that are useful for effective decision making. Daily data were collected over a period of one month and studied. A trend was then established to predict the existing system's performance. Using the means of the data as predictors, an improved model is proposed in this paper and simulated to validate the design performance.

An analysis the existing system shows that the data throughput efficiency of IEEE 802.11 decreases rapidly with the data rate, due to the nature of its design. The fundamental reason for this inefficiency is that current MAC allocates the entire channel to one station as a single resource. This allocation strategy can become too coarsegrained as the data rate increases. Even if a sender wishes to transmit small amount of data, it still needs to contend for the entire channel. Such contention resolution time posses serious overhead to the channel time used for data transmission. Thus, as the data rate increases, the throughput efficiency extends the useful channel time for data transmissions by sending multiple frames together in one contention period (frame aggregation). This larger aggregated frame results in longer delays as the sender must wait to receive enough frames before actual transmission, which adversely affects TCP real-time applications and even web browsing that involves chatty protocol or short-lived sessions. It is expected that our fine-grained channel access model will improve WLAN efficiency by effectively reducing the channel width and create more channels, where the channel width is commensurate with the data rate and typical frame size. Multiple stations or network classes can then contend for and use these smaller channels simultaneously according to their traffic demands, thereby amortizing MAC coordination and increasing the overall efficiency.

\section{The system model}

Current IEEE 802.11 WLANs use carrier sensing multiple access with collision avoidance (CSMA/CA) for their MAC protocol. The MAC protocol employs a random backoff scheme to avoid the simultaneous transmission of multiple nodes. In order to build a simple model for the network efficiency ratio of IEEE 802.11 WLANs, we require important inter-frame data that play key role in message transmission. These parameters include:

Shortest inter-frame space (SIFS): This is the shortest time interval between the data frame and its acknowledgement (i.e., the least time a receiver needs to return a message to the sender), and is used for highest priority transmissions to enable prioritized stations gain immediate access to the radio link. This parameter is given as [21]:

$t_{s i f s}=t_{r f-\text { delay }}+t_{\text {proc }}+t_{T_{x} R_{x}}$

where

$t_{r f \text {-delay }}$ is the delay incurred when transferring digital signals from the RF antenna to the processing unit

$t_{\text {proc }}$ is the time the processor requires to service incoming signals

$t_{T_{x} R_{x}}$ is the time required for the RF front-end to switch from receiving to transmit mode 
Distributed Inter-Frame Space (DIFS): This parameter is determined from SIFS and the backoff slot time, and is given as:

$t_{d i f s}=t_{s i f s}+2 . t_{s l o t}$

where

$t_{\text {slot }}$ represents the slot time and is given as

$t_{\text {slot }}=t_{c e s}+t_{T_{x} R_{x}}+t_{\text {proc }}+t_{\text {max }_{-} \text {dist }}$

where

$t_{c e s}$ is the time taken by a node to measure the channel energy that decides the channel status

$t_{\text {max_dist }}$ is the time the radio signal takes to travel the maximum distance in the network.

The various values of these parameters for the different 802.11 standards are given in Table 1:

Table 1. Inter-frame data for 802.11 standards

\begin{tabular}{c|c|c|c}
\hline Standard & $\begin{array}{c}\text { Slot time } \\
(\mu s)\end{array}$ & DIFS $(\mu s)$ & SIFS $(\mu s)$ \\
\hline IEEE 802.11b & 20 & 50 & 10 \\
IEEE 802.11a & 9 & 34 & 16 \\
IEEE 802.11g & 9 or 20 & 28 or 50 & 10 \\
\hline
\end{tabular}

From these inter-frame parameters, we deduce the efficiency-ratio of CSMA/CA, where a node selects a random number uniformly from a contention window $[0$, $\mathrm{CW}$ ), and the expected number of backoff slots $W=\frac{C W}{2}$, as:

$$
W L A N_{\text {eff_ratio }}=\frac{t_{\text {data }}}{t_{\text {slot }} \cdot W+t_{\text {diss }}+t_{\text {frame }}+t_{\text {siss }}+t_{\text {ack }}+t_{\text {data }}}
$$

where $t_{\text {data }}$ is the data transmission time

$t_{\text {frame }}$ is the time taken to transmit a frame of training symbols

$t_{\text {ack }}$ is the time taken to acknowledge a transmitted packet $W$ is the average backoff slots.

Only $t_{\text {data }}$ is used for transmitting application data, the other time parameters are simply over-heads and are constrained by physical laws and current constraints in stateof-the-art radio electronics devices. In IEEE 802.11, every data frame has a total long frame transfer time $t_{\text {frame }}$ (also referred to as preamble transfer time) of $192 \mu \mathrm{s}$. Most distributed contention resolution protocols use contention windows to control the channel access of nodes [22]. Contention windows do not only reduce network congestion, but also directly affect the share of bandwidth a node achieves during competition for the channel. The contention window for a class $C_{i}$ is given as [23]:

$$
C W_{\min }(i)=C W_{\min }(1) \frac{r_{1}}{r_{i}}
$$

where

$r_{i}$ is the bit rate of class $C_{i}$.

Precisely, in this paper, we study ten network stations of different classes. Using equation (5) guarantees for instance, that the average backoff counter timer of a given class, say $C_{1}$ is half of that of class $C_{2}$. Hence, we ensure that class $C_{1}$ stations access the medium twice as much than $C_{2}$ stations.

Having obtained throughput performance data from an IEEE $802.11 \mathrm{~b}$ wireless environment, we introduce a relationship that relates the data transmission time and the throughput thus,

$$
t_{\text {data }}=\frac{1}{T_{n}}
$$

where $T_{n}$ is the data throughput at node $n$

Substituting equation (5) into equation (4) yields:

$$
W L A N_{\text {eff_ratio }}=\frac{T_{n}^{-1}}{t_{\text {slot }} \cdot W+t_{\text {diss }}+t_{\text {frame }}+t_{\text {siss }}+t_{\text {ack }}+T_{n}^{-1}}
$$

Our finite-grained model accepts same values of $t_{\text {sifs }}$ and $t_{\text {difs }}$ of the IEEE 802.11 model. To transmit a frame of training symbol, we require three OFDMA symbols for $1 \mathrm{x}$ and 2x MIMO and four symbols for 4x MIMO. Using a 3symbol frame rate for instance, $t_{\text {frame }}=46.8 \mu \mathrm{s}$, and adding another OFDM symbol for the acknowledgement, i.e., $t_{a c k}=15.6 \mu \mathrm{s}$, the total MAC overhead becomes $157.8 \mu \mathrm{s}$. Modifying equation (4) for this purpose, we arrive at the fine-grained access efficiency ratio:

$$
F G A_{\text {eff_ratio }}=\frac{T_{n}^{-1}}{t_{\text {difs }}+t_{R T S / C T S / A C K}+t_{\text {frame }}+S y m \cdot t_{\text {sifs }}+T_{n}^{-1}}
$$

where

$t_{\text {RTS /CTS / ACK }}$ is the total time for the request-to-send, clear-to-send and acknowledgement process and is given as:

$$
t_{R T S / C T S / A C K}=t_{M-R T S}+t_{M-C T S}+t_{M-A C K}
$$

and

Sym represents the symbol frame rate

We also establish a relationship between the number of nodes $n$ and the average network delay as [24]:

$$
D_{n}=\frac{1}{1-\frac{1}{n e^{n / c}}}
$$

where

$D_{n}$ is the average network delay

Thus, the achievable throughput becomes:

$T_{n}=\frac{1-\frac{1}{n e^{n / c}}}{n}=\frac{1}{n D_{n}}$ 
To establish a relationship between the network-efficiency ratio and delay for WLAN 802.11 and fine-grained network models, we finally substitute equation (11) into equations (7) and (10) respectively, and obtain:

$$
W L A N_{\text {eff_ratio }}=\frac{n D_{n}}{t_{\text {slot }} \cdot W+t_{\text {difs }}+t_{\text {frame }}+t_{\text {sifs }}+t_{\text {ack }}+n D_{n}}
$$

and

$$
F G A_{\text {eff_ratio }}=\frac{n D_{n}}{t_{\text {diss }}+t_{\text {RTS /CTS /ACK }}+t_{\text {frame }}+S y m \cdot t_{\text {sis }}+n D_{n}}
$$

\section{Models simulation and discussion of results}

The field data were used to test the performance of both models and more simulation runs were carried out to ascertain the evaluation. The input parameters and the respective values are shown in Table 2. These inputs on the average gave optimum performance and enabled us report on the models' performance.

\begin{tabular}{|c|c|}
\hline Parameter & Value \\
\hline Data rate $\left(T_{n}\right)$ & $\begin{array}{l}\text { Inbound }(\mathrm{kb} / \mathrm{s}): 3.351,1.624,96.056,151.693 \text {, } \\
78.122,23.476,10.057,7.101,4.667,2.422 \\
\text { Outbound }(\mathrm{kb} / \mathrm{s}): 25.113,70.558,41.187,25.729, \\
15.048,9.478,5.482,2.946,2.190,1.597\end{array}$ \\
\hline $\begin{array}{l}\text { Distributed Inter-Frame Space } \\
\left(\mathrm{t}_{\text {difis }}\right)\end{array}$ & $50 \mu \mathrm{s}$ for both systems \\
\hline $\begin{array}{l}\text { Shortest Inter-Frame Space } \\
\left(\mathrm{t}_{\text {sifs }}\right)\end{array}$ & $10 \mu$ s for both systems \\
\hline Number of backoff slots (W) & $\mathrm{W} 1=3.0, \mathrm{~W} 2=7.0, \mathrm{~W} 3=15.0, \mathrm{~W} 4=31.0$ \\
\hline Number of nodes (n) & $20-200$ \\
\hline Number of Network class (c) & $100-1000$ \\
\hline Slot time $\left(t_{\text {slot }}\right)$ & ${ }^{20} \mu s$ \\
\hline $\begin{array}{l}\text { Frame transmit time }\left(\mathrm{t}_{\text {frame }}\right) \\
\text { Time taken to acknowledge a } \\
\text { transmitted packet }\left(\mathrm{t}_{\text {ack }}\right)\end{array}$ & $\begin{array}{l}\text { IEEE WLAN }=192, \text { F-GA }=46.8 \\
38 \mu s\end{array}$ \\
\hline $\begin{array}{l}\text { Total time for the request-to- } \\
\text { send, clear-to-send and } \\
\text { acknowledgement process }\left(\mathrm{t}_{\mathrm{rca}}\right)\end{array}$ & $157.8 \mu s$ \\
\hline Symbol frame rate (sym) & 3 \\
\hline
\end{tabular}

Table 2. Simulation models parameters

Sample outputs were generated in the form of graphs and used to discuss the behavior of the proposed system models as follows:

Figures 1(a) and 1(b) relate the network efficiency as a function of data rate for IEEE 802.11 WLAN inbound and outbound traffics. It is observed from these graphs that as the data rate increases, the efficiency decreases exponentially for both traffics. But the efficiency seems improved in the outbound than inbound traffic. The efficiency is further inhibited by the number of backoff slots or the size of the contention window, i.e., the efficiency decreases as the number of backoff slots increases. Therefore, in order to improve the performance in practice, careful selection of the backoff slots should be made.

In Figures 2(a) and 2(b), graphs of network efficiency and delay for IEEE WLAN inbound and outbound traffics are shown. From the graphs, the efficiency decreases with increase in delay. This is caused by the extension of useful channel time for data transmissions through the transmission of larger frames. The larger aggregated frame causes longer delays as the sender has to wait to accumulate enough frames, before actual transmission. The number of backoff slots also contributes to dropping the network efficiency. However, there exists not much significant difference in performance drop (considering both traffics).
Figures 3(a) and 3(b), show the graphs of network efficiency vs. number of nodes for IEEE WLAN inbound and outbound traffics. From these graphs, the efficiency of the system degrades as the number of backoff slots is increased and the rate of degradation is determined by the growth in the number of users that access the network.

Figures 4(a) and 4(b) show the graphs of efficiency vs. data rate, using the fine-grained model, for both inbound and outbound traffics. Here, as the data rate increases, the system efficiency drops for both traffics. Comparing these to Figures 1(a) and 1(b), we observe an improved performance over the WLAN Model. This improvement is as a result of additional parameters introduced to model the data transfer speed, by allowing each node autonomous access to the pool of resources.

Figures 5(a) and 5(b) relates the network efficiency and delay for fine-grained inbound and outbound traffics. We observe here that for both traffics, the system efficiency also degrades as the delay increases. But there is a significant performance improvement over the IEEE 802.11 WLAN.

In Figures 6(a) and 6(b), the graphs of data rate vs. delay for inbound and outbound traffics are presented. In both graphs, the data rate of the system decreases with increase in delay. The observed pattern could be attributed to some delay-sensitive traffic applications (e.g., video conferencing or web browsing). These operations contribute to severe network aggregation, but could be controlled using the fine-grained model.

Figures 7(a) and 7(b) show plots of data rate vs. number of nodes for inbound and outbound traffics. In both graphs, the data rate increases with number of nodes, but care must be taken by network operators to control the number of nodes that could cause system saturation and impact negatively on the systems performance

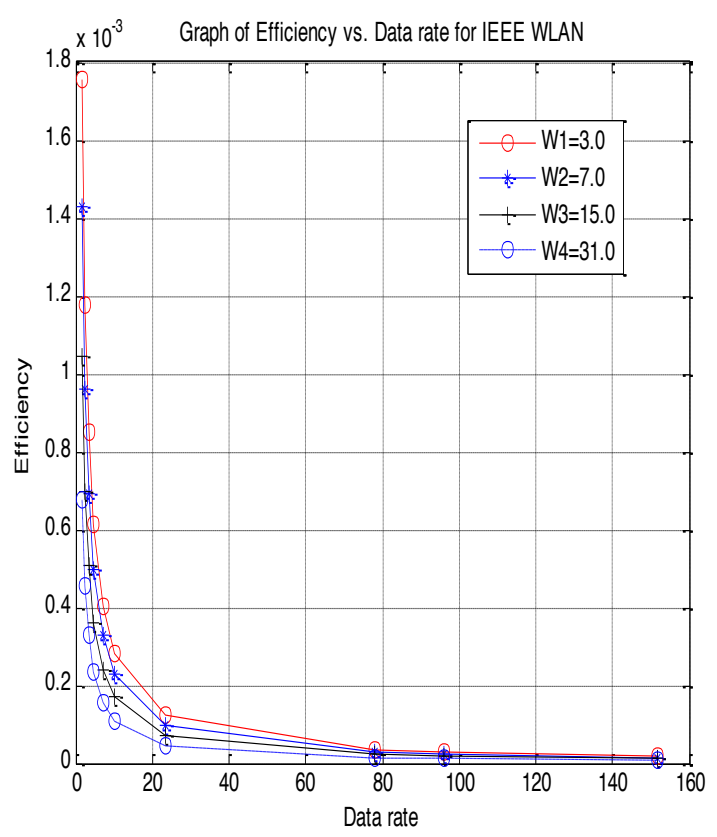

Fig. 1. (a) Graph of Efficiency vs. Data rate for IEEE WLAN Inbound. 


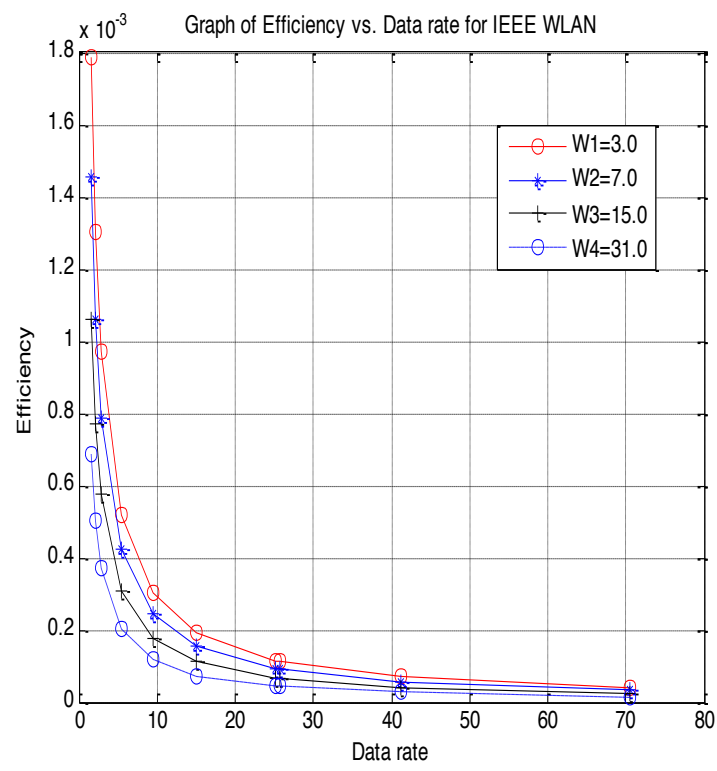

Fig. 1. (b) Graph of Efficiency vs. Data rate for IEEE WLAN Outbound.

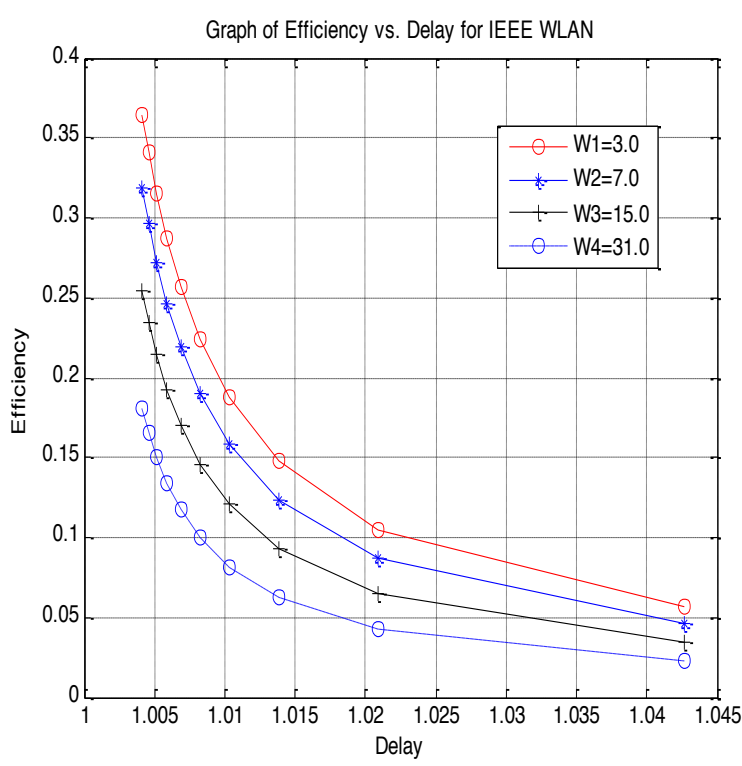

Fig. 2. (a) Graph of Efficiency vs. Delay for IEEE WLAN Inbound

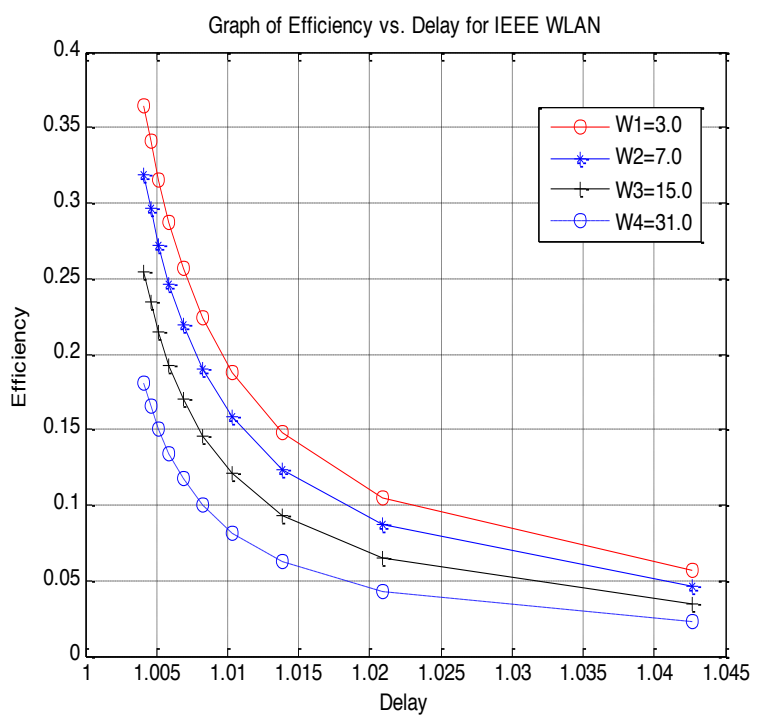

Fig. 2. (b) Graph of Efficiency vs. Delay for IEEE WLAN Outbound

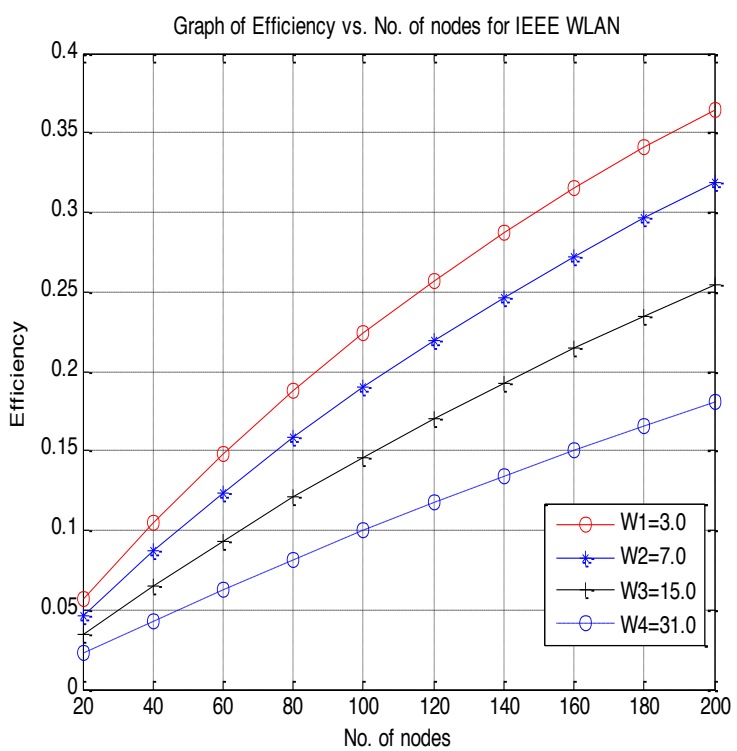

Fig. 3. (a) Graph of Efficiency vs. No. of nodes for IEEE WLAN Inbound

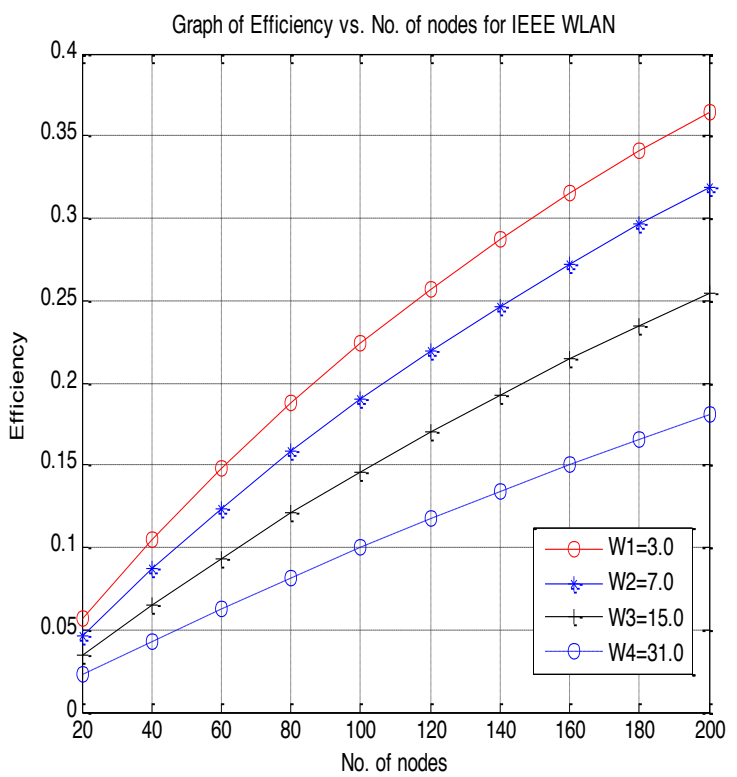

Fig. 3. (b) Graph of Efficiency vs. No. of nodes for IEEE WLAN Outbound

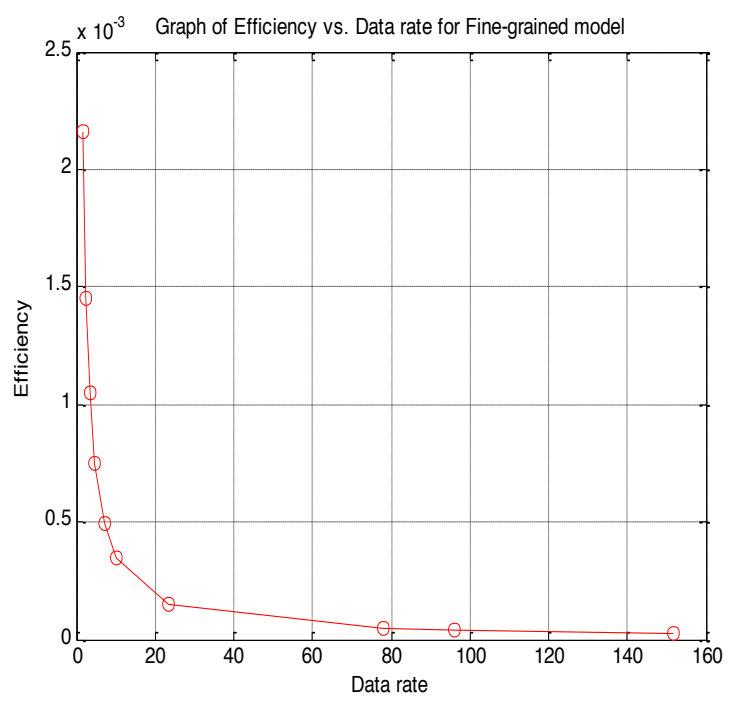

Fig. 4. (a) Graph of Efficiency vs. Data rate for fine-grained model Inbound 


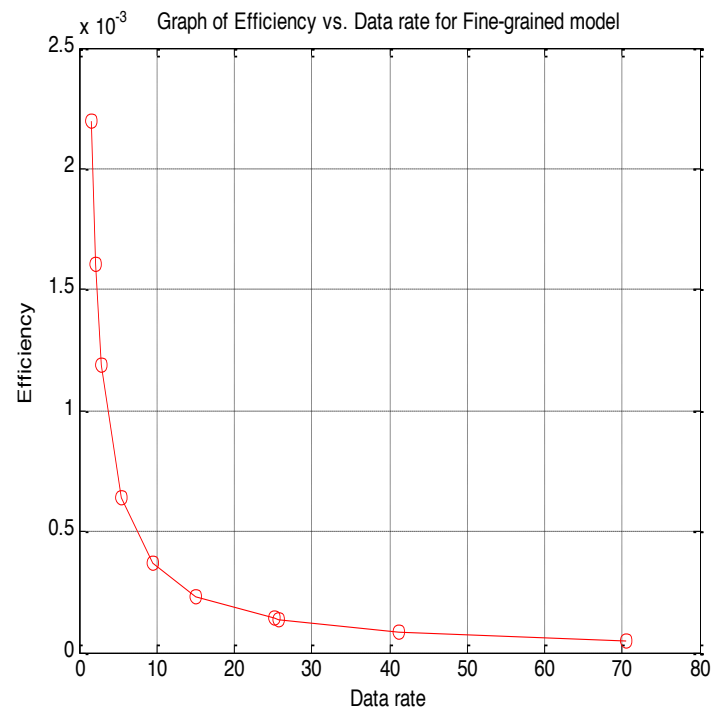

Fig. 4. (b) Graph of Efficiency vs. Data rate for fine-grained model Outbound

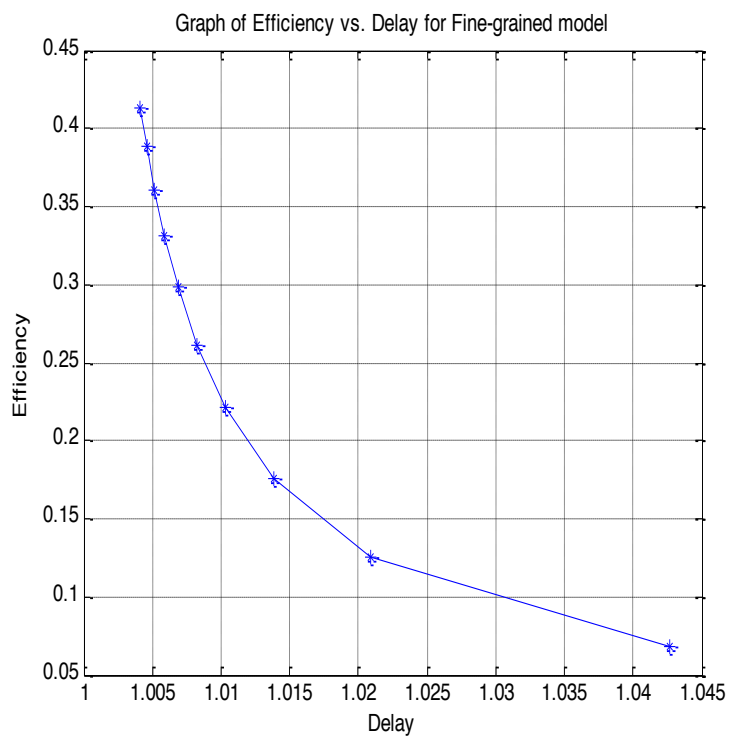

Fig. 5. (a) Graph of Efficiency vs. Delay for fine-grained model Inbound

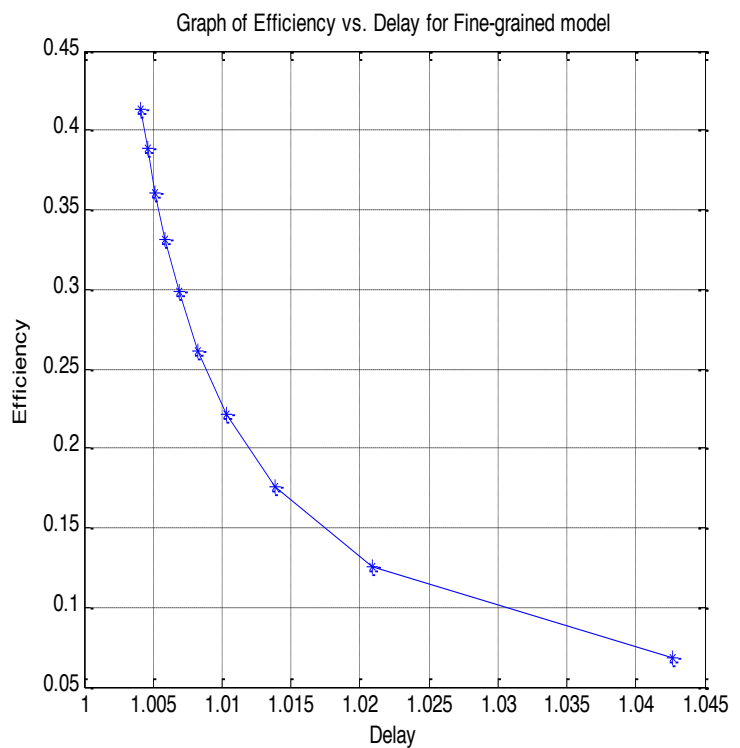

Fig. 5. (b) Graph of Efficiency vs. Delay for fine-grained model Outbound

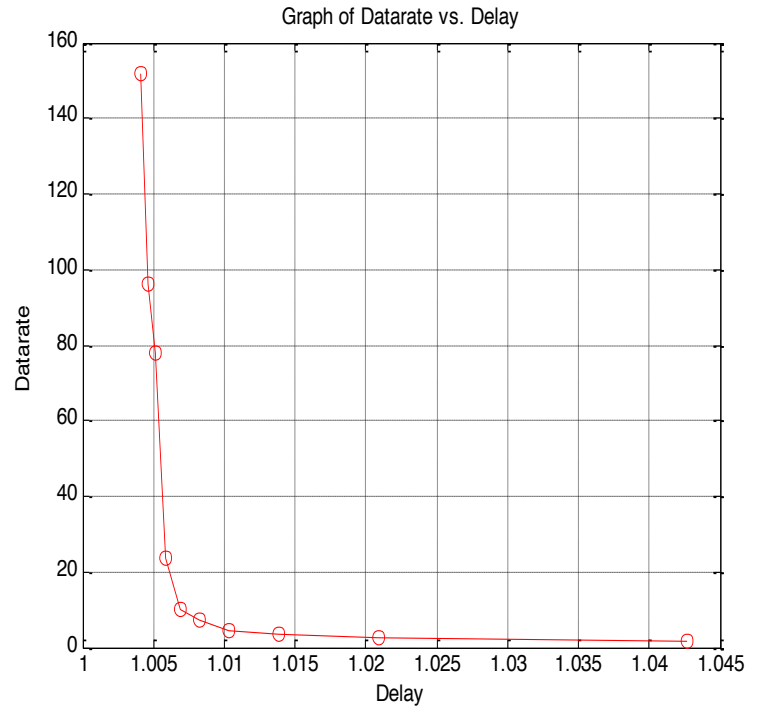

Fig. 6. (a) Graph of Data rate vs. Delay - Inbound

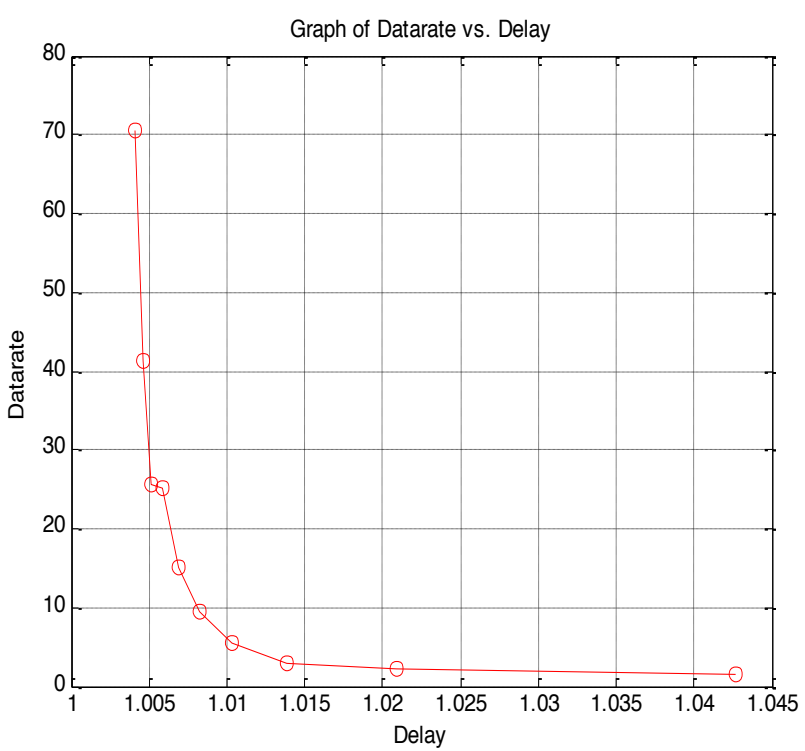

Fig. 6. (b) Graph of Data rate vs. Delay - Outbound

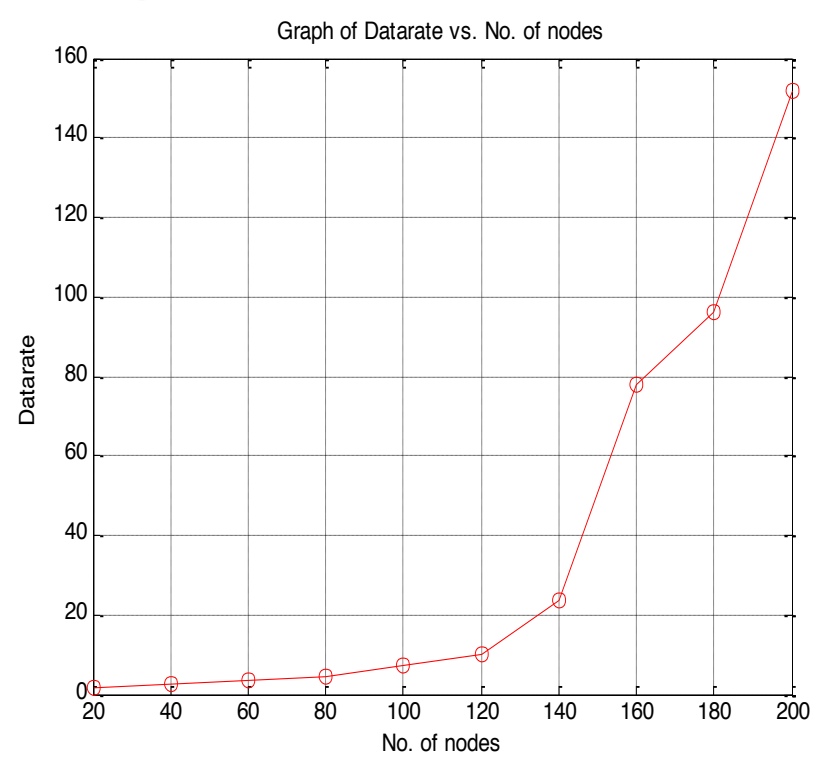

Fig. 7. (a) Graph of Data rate vs. No. of nodes - Inbound 


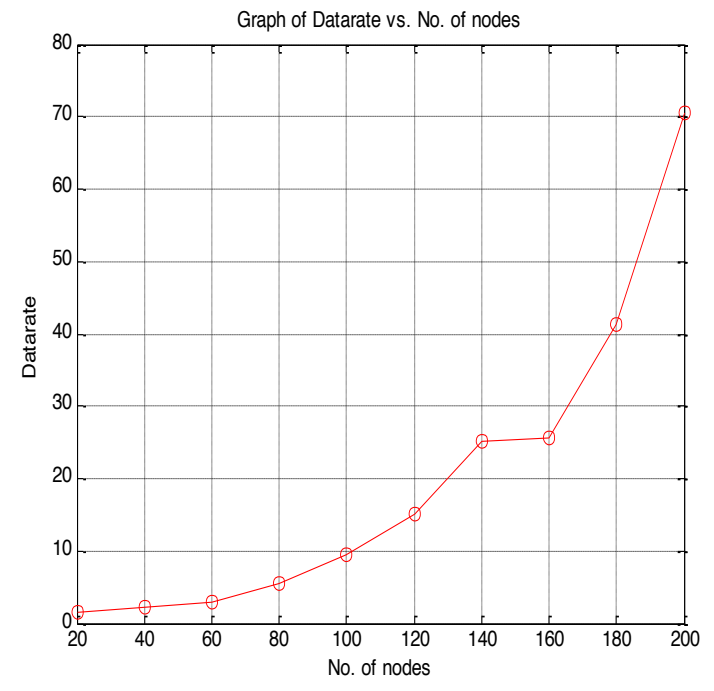

Fig. 7. (b) Graph of Data rate vs. No. of nodes - Outbound

\section{Conclusion}

We have identified the various problems associated with network efficiency in IEEE 802.11 WLAN systems and have proposed a fine-grained approach improves the network performance. We observed that the fine-grained approach improved channel access by effectively reducing the width of channels, thus, enabling more users' access to the network, as well as increasing the throughput of the entire system.

This paper considered the optimization of network efficiency. The goal was to improve WLAN channel access through a study of an existing WLAN environment. Empirical data were collected from the field, and the proposed models analyzed through extensive computer simulations. Relationships were also established between the various system parameters to enable an effective interpretation of the systems' performance.

\section{References}

1. Shrivastava, V., Rayanchu, S., Yoon, J., and Banerjee, S.: $802.11 \mathrm{n}$ under the microscope. In: Proceedings of $8^{\text {th }}$ ACM SIGCOMM on Internet Measurement Conference (IMC), Vouliagmeni, Greece, 2008, pp. 105-109.

2. Prakash, G. and Thangaraj, P.: Analytical Modeling of IEEE 802.11e Enhanced Distributed Channel Access under a Nonstauration Condition. Journal of Computer Science. 7(4) 554-560 (2011).

3. Salyers, D. and Striegel, A.: A Novel Approach for Transparent Bandwidth Conservation. In: Proceedings of IFIP Networking: 1219 - 1230 (2005).

4. Chesire, M., Wolman, A., Voelker, G. and Levy, A.: Measurement and Analysis of a streaming media workload. In USENIX symposium on Internet Technologies and Systems (USITS): 7 - 12 (2001).

5. Park, S. and Park, D.: Adaptive Core Multicast Routing Protocol. Wireless Networks, 10(1) 53-60 (2004).

6. Badrinath, B. and Sudame, G.: Gathercast: The design and implementation of a programmable aggregation mechanism for the Internet. In Proceedings of IEEE ICCCN, Las Vegas, NV USA, 2000, pp. 206-213.

7. Iyer, S., Zhang, R. and McKeown, N.: Routers with a single stage of buffering, In: In Proceedings of SIGCOMM 2002, New Yorks, NY, USA, ACM Press: 251-264, (2002).

8. Yun, K. I.: A terabit multiservice switch. IEEE Micro, 21(1) 5870, (2001).

9. Floyd, S.: High-speed TCP for large congestion windows, Network Working Group, Internet Society (2003).

10. Bernat, G. and Burns, A.: Combining (mn). Hard Deadlines and Dual Priority Scheduling. In: Proceedings of the $18^{\text {th }}$ IEEE RealTime Systems Symposium, San Francisco, CA: 46-57 (1997).

11. Hua, S., Qu, G. and Bhattacharyya, S.: Energy Reduction Techniques for Multimedia Applications with Tolerance to Deadline Misses. In Proceedings of the $4^{\text {th }}$

12. Conference on Design Automation: 131-136 (2003).

13. Hamdaoui, M. and Ramanathan, P.: A Dynamic Priority Assignment Technique for Streams with (M,K)- Firm Deadlines. IEEE Transactions on Computing. 44(12) 1443-1451, (1995).

14. Hua, S. and Qu, G.: Energy-efficient Dual Voltage soft RealTime System with $(\mathrm{m}, \mathrm{k})$ firm Deadline Guarantee. In Proceedings of International Conference on Compilers
Architecture and Synthesis for Embedded Systems:, Washington, DC: 116-123 (2004).

15. Lindsay, W. and Ramanathan, P.: A Technique for Meeting endto-end $(\mathrm{m}, \mathrm{k})$ guarantee requirements in point-to-point networks. In: Proceedings of the $22^{\text {nd }}$ Annual IEEE conference on Local Computer Networks Minneapolis, MN: 294-305 (1997).

16. Zhang, Y. and West, R.: End-to-end window constrained scheduling for real-time communication. In: Proceedings of the $10^{\text {th }}$ International Conference on Real-Time and Embedded Computing Systems and Applications (RTSCA), Gothenburg, Sweden: 1-20 (2004).

17. Gutwin, C., Fedak, C., Watson, M., Dyck, J. and Bell, T.: Improving Network Efficiency in Real-Time Groupware with General Message Compression. In proceedings of ACM CSCW conference, Banft, Alberta, Canada: 119-128 (2006)

18. Ziv, J. and Lempel, A.: A Universal Algorithm for Sequential Data Compression, IEEE Transactions on Information Theory: 337-343 (1977).

19. Salomon, D.: A Concise Introduction to Data Compression. Springer-Verlag Limited, London (2008).

20. Nagurney, A.: A Network Efficiency Measure for Congested Networks. Europhysic, Letters 71 (2007): 1-5 (2007).

21. Latora, V. and Merchiori, M.: Efficient Behaviour of smallworld Networks. Phys. Rev. Lett. 87 (Article No. 198701) (2001).

22. Burton, M.: 802.11 Arbitration: White Paper (Version 1.00). Certified Wireless Network Professionals (CWNP), Inc., Atlanta GA, USA (2009).

23. Yang, Y., Wang, J. and Kravets, R.: Distributed Optimal Contention Window Control for Elastic Traffic in Wireless LANs. In proceedings of 24th Annual Joint Conference of the IEEE Computer and Communications Societies: 1-12 (2005).

24. Chetoui, Y. and Bouabdallah, N.: Adjustment mechanism for the IEEE 802.11 contention window: An efficient bandwidth sharing scheme. Computer Communications, 30 (2007): 2686-2695 (2007).

25. Kuo, J-C., Liao, W. and Hou, T. C.: Impact of Node Density on Throughput and Delay Scaling in Multi-Hop Wireless Networks. IEEE Transactions on Wireless Communications. 8(10): 51035111 (2009). 